\title{
Breastfeeding rates and duration in Germany: a Bavarian cohort study
}

\author{
Martina Kohlhuber ${ }^{1 *}$, Barbara Rebhan ${ }^{1}$, Ursula Schwegler ${ }^{1}$, Berthold Koletzko ${ }^{2}$ and Hermann Fromme ${ }^{1}$ \\ ${ }^{1}$ Department of Environmental Health, Bavarian Health and Food Safety Agency, Veterinaerstrasse 2, 85764 Oberschleissheim, \\ Germany \\ ${ }^{2}$ Dr von Hauner Children's Hospital, Ludwig Maximilian University of Munich, Lindwurmstrasse 4, 80337 Muenchen, Germany \\ (Received 25 July 2007 - Revised 8 October 2007 - Accepted 12 October 2007 - First published online 25 February 2008)
}

Breastfeeding is the recommended feeding for all healthy infants. The aim of our study was to assess the current state of breastfeeding prevalence, duration and behaviour in Bavaria, Germany as a basis for targeting breastfeeding promotion measures. The Bavarian Breastfeeding Study is a prospective cohort study of 3822 mothers who delivered in April 2005 in Bavaria, Germany. Breastfeeding duration and determinants such as socioeconomic status, attitudes towards breastfeeding, birth mode and breastfeeding problems were assessed by questionnaires $2-6 \mathrm{~d}$ after birth and 2, 4, 6, and 9 months after birth. The initial breastfeeding rate was $90 \%$. After 4 months $61 \%$ still breastfed (any breastfeeding). In the multivariate analyses the main influencing factor reducing breastfeeding initiation was the partner's negative attitude towards breastfeeding (OR 21.79; $95 \%$ CI 13.46, 35.27). No initial breastfeeding was also associated with lower education, maternal grandmother's negative attitude and pre-term birth. Protective factors were primary breastfeeding experience and information on breastfeeding before birth. Breastfeeding duration $<4$ months was strongly associated with breastfeeding problems (OR 7.56; $95 \%$ CI 6.21, 9.19), smoking, lower education, partner's negative attitude and Caesarean section. Since the attitude of family members is an important influencing factor on breastfeeding rates, breastfeeding promotion should also target the partners of pregnant women and the families of newborn infants. Public health interventions such as more effective support for the management of breastfeeding problems, especially in lower social status families, should be implemented and their effectiveness should be critically evaluated.

Breastfeeding: Infant nutrition: Breast milk

Breastfeeding is the recommended feeding for healthy infants. Three recent meta-analyses indicate that breastfeeding protects against gastrointestinal and to a lesser extent also other infections and provides a variety of other health benefits ${ }^{(1-3)}$, including a reduced risk of later overweight or obesity ${ }^{(4,5)}$.

In 1981 breastfeeding initiation rates in two German cities were between 87 and $95 \%$, and only $2-6 \%$ of infants were still fully breastfed at 4 months of age ${ }^{(6)}$. The main reason why mothers ceased breastfeeding early was breastfeeding problems. In 1997 breastfeeding initiation rates were reported as about $91 \%{ }^{(7)}$, with $44 \%$ of the infants still fully breastfed at 4 months.

The WHO recommends exclusive breastfeeding for 6 months as a goal for populations, and continued breastfeeding thereafter ${ }^{(8)}$. The German National Breastfeeding Committee recommends exclusive breastfeeding for 6 months and continued partial breastfeeding as long as mother and child wish ${ }^{(9)}$.

The aim of our study was to assess the current state of breastfeeding prevalence, duration and behaviour in Bavaria in a prospective study to provide a basis for developing targeted breastfeeding promotion measures.

\section{Methods}

The Bavarian Breastfeeding Study is a prospective cohort study planned as a survey of all mothers who delivered in the state of Bavaria, southern Germany, in April 2005. All Bavarian birth clinics, birth houses and midwives who assisted mothers in home birth were asked to participate as project partners to recruit participants. The basic questionnaire was distributed by the project partners in April 2005 2-6d after birth to mothers who delivered a baby in the participating clinics, birth houses or at home. The questionnaire was available in German and Turkish language. There were no exclusion criteria, except for being $<18$ years old due to local data protection and consent regulations. Mothers of twins were told to answer the questionnaire for the first-born child. The study protocol was reviewed by the Ethics Committee of the Bavarian Medical Association, and written consent was obtained from all participants.

A questionnaire was sent by mail to participating mothers at 2 months after birth. To limit costs, only mothers who indicated in the previous questionnaire that they still breastfed received a questionnaire at 4 and 6 months after birth. However, at 9 months after birth all participants who initially participated were again asked to fill in a questionnaire. We tried to enhance participation rates by asking paediatricians to distribute questionnaires in their offices to mothers who delivered in April 2005 and by placing advertisements in the local press. All participants who did not send in questionnaires within 4 weeks were contacted by telephone to remind them to fill in

* Corresponding author: Martina Kohlhuber, fax +49 8931560 835, email Martina.Kohlhuber@lgl.bayern.de 
the questionnaire, or if they were not willing to do so, to participate in a short telephone interview to get basic information. Breastfeeding was defined as follows:

Exclusive breastfeeding: only breast milk, no other liquids or infant formula;

Full breastfeeding: breast milk plus liquids like tea or water (no infant formula);

Partial breastfeeding: breast milk plus other feeds such as liquids, infant formula, complementary food.

Breastfeeding determinants were socioeconomic status which was assessed as mother's highest educational degree and grouped in four categories: no educational degree; low ( $<10$ years); medium (10-11 years) and high education $(>11$ years). Family status was assessed by the question 'Are you living together with your partner?' (Yes/no). Smoking status was assessed in the baseline questionnaire (2-6 d after birth) asking 'Do you smoke currently?' and was grouped into 'no' and 'yes (occasionally or regularly)'.

Partner's and grandmother's attitudes were asked by 'Which attitude towards breastfeeding has your partner/mother/your partner's mother?' Answers were grouped binary as positive ('He/she thinks it's good or important') and negative ('He/she doesn't want me to breastfeed/doesn't know/I don't know his/ her attitude'). Breastfeeding experience was asked as 'Did you breastfeed your last child?' (Yes/no). Pre-term birth is defined as born before the 37th week of pregnancy. Birth mode was grouped into vaginal delivery, Caesarean section and vaginal surgery (forceps, ventouse, breech delivery). No open questions were used for this analysis.

Regarding representation we compared the characteristics of the participants with statistics from the Bavarian State Office for Statistics. For statistical analyses we used SPSS 13.0. Breastfeeding rates were computed considering loss to follow up. Bivariate analyses were performed by $\chi^{2}$ tests. In multivariate analyses we computed OR and $95 \%$ CI. Variables not significant in the bivariate analyses were omitted from the multivariate models.

\section{Results}

Of the 146 birth clinics in Bavaria, 141 participated as project partners in the study, two of them being baby-friendly hospitals. Ten birth houses and thirty midwives who support mothers in home birth also distributed questionnaires. Of the 8805 mothers who delivered in April 2005, some 3840 completed a questionnaire in the baseline survey $(43.6 \%)$. Eleven mothers were excluded because they were younger than 18 years old. Three participants withdrew their written consent later. Four questionnaires were excluded because they were filled in incorrectly or incompletely. Therefore 3822 valid questionnaires are included in the basic survey. The further follow-up rates range between 82 and $97 \%$ (Table 1).

\section{Characteristics of the study population}

The average age of participating mothers was 31.7 (range 18-46) years. The participating mothers were older than the average age of mothers who delivered in 2005 (data regarding proportionality are shown in Table 2). The percentage of the mothers who were not born in Germany was 15.9. Most of
Table 1. Participation and follow-up rates

\begin{tabular}{lccc}
\hline & $\begin{array}{c}\text { Obtained } \\
\text { questionnaires } \\
(n)\end{array}$ & $\begin{array}{c}\text { Contacted } \\
\text { participants } \\
(n)\end{array}$ & $\begin{array}{c}\text { Percentage } \\
\text { follow-up rate of } \\
\text { contacted participants }\end{array}$ \\
\hline $2-6$ d after birth & 3822 & - & $43 \cdot 6^{\star}$ \\
2 months & 3370 & 3822 & $88 \cdot 1$ \\
4 months & 2562 & 2639 & $97 \cdot 1$ \\
6 months & 2255 & 2320 & $97 \cdot 1$ \\
9 months & 3103 & 3783 & 82.0 \\
\hline
\end{tabular}

*Participation rate (\%) of all mothers who delivered in Bavaria in April 2005.

them were born in Eastern European countries including the former Soviet Union. The percentage of the participants who did not live together with their partners was 4.7. The educational level of the participants was higher than the average of mothers in Bavaria. One in four participants had $<10$ years of school education, $37.2 \% 10-11$ years and $35.7 \%$ $>11$ years. Of the children $48 \%$ were female and $1.3 \%$ were twins; $27 \%$ were delivered by Caesarean section.

\section{Breastfeeding conditions}

Only $42 \%$ of the breastfeeding mothers participated in full rooming-in. Most of the mothers were instructed in the hospital by a (infant-) nurse or a midwife and only $2 \%$ by a lactation consultant. Of the breastfed infants $37 \%$ received formula or additional fluids during the first days of life.

\section{Breastfeeding rates}

Almost $90 \%$ of mothers started to breastfeed (Table 3). Two months after birth $70 \%$ still breastfed (44.7\% exclusive

Table 2. Description of the study population and of the total population of women who delivered in Bavaria in the year 2005 by proportion (\%)

\begin{tabular}{|c|c|c|}
\hline & Study population & $\begin{array}{l}\text { Women who delivered } \\
\text { in Bavaria in } 2005\end{array}$ \\
\hline Age of mother(years) & $n 3427$ & $n 107308$ \\
\hline$<20$ & 0.7 & $2 \cdot 0$ \\
\hline $20-24$ & $7 \cdot 8$ & $12 \cdot 6$ \\
\hline $25-29$ & $24 \cdot 0$ & 27.5 \\
\hline $30-34$ & $38 \cdot 1$ & 33.0 \\
\hline $35-49$ & $24 \cdot 4$ & 20.5 \\
\hline$\geq 40$ & 4.9 & $4 \cdot 3$ \\
\hline Years of education & $n 3822$ & n 107308 \\
\hline$<10$ years & 23.7 & $32 \cdot 0$ \\
\hline $10-11$ years & $35 \cdot 6$ & 33.5 \\
\hline$>11$ years & $35 \cdot 7$ & $30 \cdot 1$ \\
\hline No degree & $1 \cdot 8$ & \\
\hline Other (foreign) degree & 1.6 & 4.4 \\
\hline Missing & 1.6 & \\
\hline Country of birth/nationality & $n 3822$ & n 107308 \\
\hline Germany & $84 \cdot 1$ & $82 \cdot 3$ \\
\hline Other country & $15 \cdot 9$ & $17 \cdot 7$ \\
\hline Sex of child & & n 107308 \\
\hline Male & $51 \cdot 6$ & $51 \cdot 4$ \\
\hline Female & $48 \cdot 4$ & $48 \cdot 6$ \\
\hline Birth weight (g) & $n 3363$ & \\
\hline$<2500$ & 4.4 & $7 \cdot 1$ \\
\hline $2500-2999$ & $13 \cdot 2$ & $16 \cdot 9$ \\
\hline $3000-3499$ & $40 \cdot 9$ & $67 \cdot 0$ \\
\hline $3500-3999$ & $31 \cdot 0$ & $9 \cdot 0$ \\
\hline$\geq 4000$ & $10 \cdot 6$ & \\
\hline
\end{tabular}


Table 3. Breastfeeding rates (\%) at birth, 2, 4 and 6 months

\begin{tabular}{|c|c|c|c|c|c|}
\hline & Exclusive breastfeeding & Full breastfeeding & Partial breastfeeding & Any breastfeeding & No answer/no breastfeeding \\
\hline Initial & & 89.5 & & 89.5 & 10.5 \\
\hline 2 months & $44 \cdot 7$ & $15 \cdot 5$ & 9.5 & $69 \cdot 7$ & $30 \cdot 3$ \\
\hline 4 months & $41 \cdot 7$ & $9 \cdot 9$ & $9 \cdot 3$ & $60 \cdot 6$ & $39 \cdot 1$ \\
\hline 6 months & $21 \cdot 4$ & $4 \cdot 1$ & $26 \cdot 1$ & $51 \cdot 6$ & $48 \cdot 6$ \\
\hline
\end{tabular}

breastfeeding). After 4 months breastfeeding rates dropped to $61 \%$, after 6 months to $52 \%$.

Since a disproportionately high percentage of the loss to follow-up were participants who indicated in the baseline survey that they did not want to breastfeed we considered them in the analyses of the breastfeeding rates as participant who ceased breastfeeding (Table 3 ). When omitting the participants who did not answer all questionnaires $(n 916)$ breastfeeding rates would be overrated: 2 months, $78 \%$ v. $70 \%$; 4 months, $73 \%$ v. $61 \%$ and 6 months, $66 \%$ v. $52 \%$ (any breastfeeding).

\section{Determinants of initial breastfeeding}

In a multivariate analysis we computed adjusted $\mathrm{OR}$ and $\mathrm{CI}$ using logistic regression. Due to missing values the number of cases is reduced to $n$ 2316. The model shows OR for not breastfeeding $v$. starting to breastfeed after birth. Only variables which were significant in bivariate analyses were included in the model; child's sex and community size were therefore not included in the analyses. The main influencing factor on initial breastfeeding was the partner's attitude towards breastfeeding (Table 4). If the partner had a negative attitude, the mother had a twenty-two times higher risk not to breastfeed from the beginning (OR 21.79; $95 \%$ CI 13.46, 35.27). The maternal grandmother also had an important influence on the mother's decision to breastfeed. Mothers with $<10$ years of education were more likely not to breastfeed (OR 3.88; $95 \%$ CI 2.11, 7.12). Participants with 10-11 years of education had a higher risk not to breastfeed than women with $>11$ years of education. Pre-term birth was a risk factor for not breastfeeding. Protective factors were previous breastfeeding experience (OR $0.25 ; 95 \%$ CI 0.15 , 0.42 ) and receipt of information on breastfeeding before birth (OR 0.37; $95 \%$ CI 0.23, 0.60). No significant associations were observed for mother's age, country of birth, family status, smoking, birth mode, paternal grandmother's attitude towards breastfeeding or hospital size.

\section{Determinants of breastfeeding duration up to four months}

For the explanation of determinants of breastfeeding duration, breastfeeding was dichotomized: exclusive and full breastfeeding $v$. partial and no breastfeeding. In this model only variables which were significant in bivariate analyses were included in the model; child's sex and community size were left out of the analyses. Since information on breastfeeding and having more than one child are correlated strongly, only having more than one child was included in the analyses.

The main influencing factor on shorter breastfeeding duration was breastfeeding problems (Table 5). Mothers experiencing breastfeeding problems had a more than seven-fold higher risk to breastfeed $<4$ months (OR 7.56; $95 \%$ CI 6.21, 9.19). Twothirds of the mothers who stopped breastfeeding during the first 4 months indicated breastfeeding problems compared with $27 \%$ of mothers who still breastfed. Most mothers mentioned lack of milk, sore nipples and child's difficulties in sucking. Smoking was associated with reduced breastfeeding duration (OR 4.38; $95 \%$ CI 2.66, 7.21). Younger participants and mothers with lower education breastfed for less time than older mothers with higher educational level. The partner's and maternal grandmother's attitude towards breastfeeding was also associated with breastfeeding duration, to a lesser degree. Having more than one child was a protective factor (OR 0.74; $95 \%$ CI $0.60,0.90)$. Further significant associations were found with birth mode and country of birth. No associations were found with paternal grandmother's attitude, pre-term birth, clinic size or district.

Table 4. Determinants of no initial breastfeeding: adjusted OR and $\mathrm{Cl}$ of having tried to breastfeed after birth $v$. no breastfeeding $\dagger$

\begin{tabular}{|c|c|c|}
\hline & OR & $95 \% \mathrm{Cl}$ \\
\hline \multicolumn{3}{|l|}{ Age (years) } \\
\hline$\geq 35$ & 1 & \\
\hline$\leq 24$ & $1 \cdot 12$ & $0.48,2.64$ \\
\hline $25-34$ & $1 \cdot 23$ & $0.74,2.06$ \\
\hline \multicolumn{3}{|l|}{ Education } \\
\hline$>11$ years & 1 & \\
\hline No degree & 1.91 & $0 \cdot 14,25 \cdot 64$ \\
\hline$<10$ years & $3 \cdot 88^{\star \star \star}$ & $2 \cdot 11,7 \cdot 12$ \\
\hline $10-11$ years & $2 \cdot 27^{\star \star}$ & $1 \cdot 29,4.01$ \\
\hline \multicolumn{3}{|c|}{ Breastfeeding experience } \\
\hline No & 1 & \\
\hline Yes & $0.25^{\star \star \star}$ & $0.15,0.42$ \\
\hline \multicolumn{3}{|l|}{ Information } \\
\hline No & 1 & \\
\hline Yes & $0.37^{\star * *}$ & $0.23,0.60$ \\
\hline \multicolumn{3}{|l|}{ Partner's attitude } \\
\hline Positive & 1 & \\
\hline Negative & $21 \cdot 79^{\star \star \star}$ & $13 \cdot 46,35 \cdot 27$ \\
\hline \multicolumn{3}{|c|}{ Maternal grandmother's attitude } \\
\hline Positive & 1 & \\
\hline Negative & $3 \cdot 62^{\star \star \star}$ & $2 \cdot 26,5 \cdot 81$ \\
\hline \multicolumn{3}{|l|}{ Pre-term birth } \\
\hline No & 1 & \\
\hline Yes & $3.04^{\star *}$ & $1 \cdot 50,6 \cdot 15$ \\
\hline
\end{tabular}

Model demonstrated significant effect of the variable: ${ }^{*} P<0.05 ;{ }^{* \star} P<0.01$; ${ }^{\star * \star *} P<0.001$

†Further variables in the model with no significant effect: district, clinic size, birth mode, paternal grandmother's attitude, family status, smoking and country of birth. 
Table 5. Determinants of breastfeeding duration up to 4 months: adjusted $\mathrm{OR}$ and $\mathrm{Cl}$ of exclusive and full breastfeeding $v$. partial and no breastfeeding $\dagger$

\section{OR}

$95 \% \mathrm{Cl}$

Age (years)

$\geq 35$

$\leq 24$

$25-34$

Education

$>11$ years

No degree

$<10$ years

$10-11$ years

Country of birth

Germany

Other country

Smoking

No

Yes

드 Siblings

No
Yes

Breastfeeding problems

No

Yes

1

$3 \cdot 31^{\star \star \star}$

$1.45^{\star \star}$

$2 \cdot 16,5 \cdot 06$

$1 \cdot 16,1.83$

1

2.43

$2.52^{\star * *}$

$1.66^{\star \star \star}$

$0.86,6.84$

$1.91,3.34$

$1.33,2.09$

$1.54^{\star \star}$

$1 \cdot 15,2 \cdot 05$

1

$4 \cdot 38^{\star * *}$

$2 \cdot 66,7 \cdot 21$

1

$0.74^{\star \star}$

$0.60,0.90$

1

$7 \cdot 56^{\star * *}$

$6 \cdot 21,9 \cdot 19$

Partner's attitude

Positive

Negative

1

$2 \cdot 36^{\text {** }}$

$1.58,3.52$

Maternal grandmother's attitude

Positive

Negative

1

$1.62^{* * *}$

$1 \cdot 25,2 \cdot 08$

Birth mode

Vaginal delivery

Surgery

Caesarean section

1

$1.98^{* *}$

$1.69^{\text {** }}$

$1 \cdot 2,3 \cdot 25$

$1.36,2 \cdot 10$

Model demonstrated significant effect of the variable: ${ }^{*} P<0.05 ;{ }^{*} P<0.01$; ${ }^{\star \star \star *} P<0.001$.

†Further variables in the model, no significant effect: district, family status, pre-term birth, clinic size, paternal grandmother's attitude.

\section{Discussion}

Breastfeeding rates in Germany have increased since the first surveys in the 1980s. Compared to the last German study, the nationwide SuSe Study conducted in 1996-1997(7), initial breastfeeding rate in the present study is similar but breastfeeding rates at fourth and sixth months are clearly higher. Previous studies on breastfeeding prevalence and duration in Germany showed even lower rates ${ }^{(6,10)}$.

The comparison of the results of our study with breastfeeding rates reported in other European countries is limited by different study methods and different definitions of breastfeeding. In many countries breastfeeding is not monitored consistently ${ }^{(11)}$, and hence the WHO demands a common set of measures to assess breastfeeding practices, policies and programmes $^{(8)}$. Compared to data reported for Scandinavian countries, Switzerland and Austria ${ }^{(12,13)}$, breastfeeding rates in Bavaria are lower at all infant ages, but they are comparable with data for Italy ${ }^{(14)}$ and higher than in the UK, Spain or the Netherlands ${ }^{(13,15,16)}$.

\section{Determinants of breastfeeding prevalence and duration}

Our study confirmed some known determinants of breastfeeding initiation and duration ${ }^{(17)}$. The strongest association with breastfeeding initiation was found with the partner's attitude towards breastfeeding. Scott et al. ${ }^{(18)}$ and Littman et al. ${ }^{(19)}$ found similar results for paternal support in an Australian cohort. Similar to our results, the German SuSe study found associations between short breastfeeding duration and negative attitude of the partner in both eastern and western German states ${ }^{(20)}$. The attitude of the maternal grandmother as a predictor for initial breastfeeding was also described by Scott et al. ${ }^{(21)}$

Low socioeconomic status is known as a risk factor for breastfeeding initiation and duration in Europe and the $\mathrm{USA}^{(20,22-25)}$. In our study lower education had a significant influence on initial breastfeeding and was also associated with shorter breastfeeding duration. Similarly, maternal age did not influence breastfeeding initiation but showed a clear gradient with breastfeeding duration, which was also reported by Scott et al. ${ }^{(21)}$. Smoking did not influence breastfeeding initiation in the multivariate analysis but was a strong influencing factor on breastfeeding duration. A reason for this may be that the question for breastfeeding initiation was asked very early after delivery. The wording of the question was 'Did you try to breastfeed after birth'. It may be that many smoking mothers answered 'yes' but ceased breastfeeding in the first or second week so that smoking does not appear as an influencing factor for breastfeeding initiation. Light smoking is not considered as a contraindication for breastfeeding by the American Academy of Pediatrics ${ }^{(26)}$. Smoking mothers are advised to breastfeed since their children fare better regarding general health, respiratory illness and risk of sudden infant death syndrome when they are breastfed ${ }^{(26)}$. An explanation may be that some mothers wanted to breastfeed but also to start smoking again after birth and were concerned about potential harm to their children with breastfeeding and smoking. It is also possible that mothers gave socially preferred answers to this question and stated not to smoke even if they did. Other reviews and studies however found strong associations between initial breastfeeding and maternal smoking ${ }^{(27,17)}$. The British ALSPAC Study found that smoking influences breastfeeding duration negatively even after controlling for intention for breastfeeding ${ }^{(28)}$. Lande et al. ${ }^{(12)}$ found in Norway strong associations between smoking and breastfeeding cessation after 4 and 6 months.

Having breastfeeding problems during the first 4 months turned out to be the major influencing factor for short breastfeeding duration and breastfeeding cessation in the first 4 months, which was found to a lesser extent also in the SuSe study ${ }^{(20)}$ and in an Australian cohort ${ }^{(29)}$. Breastfeeding problems might be reduced by implementing international guidelines concerning breastfeeding instruction and breastfeeding conditions such as general support of rooming-in and no supplementation with additional formula and fluids to breast-fed infants without a medical indication ${ }^{(30)}$. It appears to us that such guidelines are not widely implemented in all hospitals in Bavaria. Information before delivery and breastfeeding experience are protective factors for breastfeeding ${ }^{(17)}$. Mothers who obtained information on breastfeeding are more interested and confident in breastfeeding $^{(27)}$. 


\section{Strengths and limitations}

The study was conducted as a prospective cohort study. Weekly breastfeeding rates and timely collection of reasons for breastfeeding cessation was possible. The risk of a recall bias is therefore low. The design was a complete cohort of all mothers who delivered in April 2005. The participation was voluntary; we therefore consider the participation rate (43\%) and follow-up rates (82-97\%) achieved as high. However, representation is limited since mothers more interested in breastfeeding and related issues would have been more likely to participate in the study. There is also an overrepresentation of older mothers and mothers with higher educational level in our sample, whereas younger mothers with lower education were more often lost to follow-up. Since these mothers breastfeed less often and for a shorter duration, breastfeeding rates may have been overestimated in this study.

\section{Conclusions for breastfeeding promotion}

Breastfeeding rates in Germany have increased in the last 10 years. However, the results of the study indicate various fields where breastfeeding promotion is still necessary. Since partners have a strong influence on mother's decision to breastfeed it appears important to motivate the mother's familial environment to support her during breastfeeding. Socially disadvantaged mothers need targeted support since breastfeeding rates are particularly low in this group and general promotion measures are often not attended by this group. Smoking mothers need assistance in smoking cessation in pregnancy and during breastfeeding to reduce infant exposure to environmental tobacco smoke.

Breastfeeding problems need to be addressed by integrative counselling of mothers during their stay in hospital and in the first weeks at home. Quality assurance in that field may lead to better results in breastfeeding duration. Centuori et al. ${ }^{(31)}$ point out that modification of hospital practices and providing the mother with guidance on positioning may be more effective than products like nipple cream to prevent sore nipples. The management and support by health care professionals can enhance a mother's self confidence in breastfeeding and thus reduce breastfeeding problems. As a result of this study an intervention study will be conducted in a particular region where hospital staff and midwives who support mothers after birth will be trained. The goal of this study is to give mothers integrative support in the hospitals and to increase breastfeeding rates and duration in this region where breastfeeding rates now are particularly low.

\section{Acknowledgements}

This study was financially supported by the Bavarian Minister for Environment, Health and Consumer Protection, Munich, Germany. Berthold Koletzko is the recipient of a Freedom to Discover Award of the Bristol Myers Squibb Foundation, New York, NY, USA. All authors declare that they have no competing financial interests.

M. K. and B. R. contributed to the conception and design of the work, the acquisition of the data, the analysis of the data and to the writing of the manuscript.

U. S., B K. and H. F. contributed to the conception and design of the work and to the writing of the manuscript.

\section{References}

1. van Rossum CTM, Büchner FL \& Hoekstra J (2006) Quantification of health effects of breastfeeding. Review of the literature and model simulation. RIVM report 350040001/ 2005. Bilthoven: RIVM.

2. Ip S, Chung M, Raman G, Chew P, Magula N, DeVine D, Trikalinos T \& Lau J (2007) Breastfeeding and Maternal and Infant Health Outcomes in Developed Countries. Evidence Report/Technology Assessment No. 153 (Prepared by Tufts-New England Medical Center Evidence-based Practice Center, under Contract No. 290-02-0022). AHRQ Publication No. 07-E007. Rockville, MD: Agency for Healthcare Research and Quality.

3. Horta BL, Bahl R, Martines JC \& Victora CG (2007) Evidence on the long-term effects of breastfeeding. Systematic reviews and meta-analyses Geneva: World Health Organization.

4. Arenz S, Ruckerl R, Koletzko B \& von Kries R (2004) Breastfeeding and childhood obesity - a systematic review. Int J Obes Relat Metab Disord 28, 1247-1256.

5. Harder T, Bergmann R, Kallischnigg G \& Plagemann A (2005) Duration of breastfeeding and risk of overweight: a meta-analysis. Am J Epidemiol 162, 397-403.

6. Kersting M, Wember T, Goddemeier T, Koester H, Wennemann J \& Schoch G (1987) Breast feeding studies 1981-1983 in 1,500 mothers in Dortmund and Haltern. III. Rates of breast feeding and duration of breast feeding in the first half year (in German). Monatsschr Kinderheilkd 135, 314-319.

7. Kersting M \& Dulon M (2002) Breastfeeding in Germany. Results of the SuSe-Study (in German). Monatsschr Kinderheilkd $150,1196-1201$.

8. World Health Organisation (2004) Infant and young child feeding: a tool for assessing national practices, policies and programmes. Geneva: WHO http://www.who.int/child-adolescenthealth/New_Publications/NUTRITION/icyf.pdf

9. Federal Institute for Risk Assessment (2004) Recommendations for Breastfeeding Duration, Press release 1 March 2004. Berlin: German National Breastfeeding Committee.

10. Bergmann RL, Bergmann KE, Lau-Schadensdorf S, et al. (1994) Atopic diseases in infancy. The German multicenter atopy study (MAS-90). Pediatr Allergy Immunol 5, Suppl. 6, $19-25$.

11. Yngve A \& Sjostrom M (2001) Breastfeeding in countries of the European Union and EFTA: current and proposed recommendations, rationale, prevalence, duration and trends. Public Health Nutr 4, 631-645.

12. Lande B, Andersen LF, Baerug A, Trygg KU, Lund-Larsen K, Veierod MB \& Bjorneboe GE (2003) Infant feeding practices and associated factors in the first six months of life: the Norwegian infant nutrition survey. Acta Paediatr 92, $152-161$.

13. Cattaneo A, Yngve A, Koletzko B \& Guzman LR (2005) Protection, promotion and support of breast-feeding in Europe: current situation. Public Health Nutr 8, 39-46.

14. Giovannini M, Riva E, Banderali G, Scaglioni S, Veehof SHE, Sala M, Radaelli G \& Agostoni C (2004) Feeding practices of infants through the first year of life in Italy. Acta Paediatr 93, 492-497.

15. Lozano de la Torre MJ, Martín-Calama J \& Hernandez-Aguilar MT (2001) Breast-feeding in Spain. Public Health Nutr 4, $1347-1351$.

16. Bulk-Bunschoten AMW, van Bodegom S, Reerink JD, Pasker-de Jong PCM \& de Groot CJ (2001) Reluctance to continue breastfeeding in The Netherlands. Acta Paediatr 90, 1047-1053.

17. Yngve A \& Sjostrom M (2001) Breastfeeding determinants and a suggested framework for action in Europe. Public Health Nutr 4, 729-739. 
18. Scott JA, Binns CW \& Aroni RA (1997) The influence of reported paternal attitudes on the decision to breast-feed. J Paediatr Child Health 33, 305-307.

19. Littman H, VanderBrug Medendorp S \& Goldfarb J (1994) The decision to breastfeed: the importance of father's approval. Clin Pediatrics 33, 214-219.

20. Dulon M, Kersting M \& Schach S (2001) Duration of breastfeeding and associated factors in Western and Eastern Germany. Acta Paediatr 90, 931-935.

21. Scott JA, Landers MCG \& Binns CW (2001) Factors associated with breastfeeding at discharge and duration of breastfeeding. J Paediatr Child Health 37, 254-261.

22. Bertini G, Perugi S, Dani C, Pezzati M, Tronchin M \& Rubaltelli FF (2003) Maternal education and the incidence and duration of breast feeding: a prospective study. J Pediatr Gastroenterol Nutr 37, 447-452.

23. Wolf JH (2000) Low breastfeeding rates and public health in the United States. Am J Public Health 93, 2000-2010.

24. Heck KE, Braveman P, Cubbin C, Chavez GF \& Kiely JL (2006) Socioeconomic factors and breastfeeding initiation among Californian mothers. Public health report 121, 51-59.
25. Noble S, ALSPAC Study Team (2001) Maternal employment and the initiation of breastfeeding. Acta Paediatr 90, $423-428$.

26. Lawrence RA \& Lawrence RM (2005) Breastfeeding: a Guide for the Medical Profession. Philadelphia: Elsevier Mosby.

27. Dennis CL (2002) Breastfeeding initiation and duration: a 1990-2000 literature review. J Obstet Gynecol Neonatal Nurs 31, $12-32$.

28. Donath SM \& Amir LH (2003) Relationship between prenatal infant feeding intention and initiation and duration of breastfeeding: a cohort study. Acta Paediatr 92, 352-356.

29. Scott JA, Binns CW, Oddy WH \& Graham KI (2007) Predictors of breastfeeding duration: evidence from a cohort study. Pediatrics 117, e646-e655.

30. Merten S, Dratva J \& Ackermann-Liebrich U (2005) Do babyfriendly hospitals influence breastfeeding duration on a national level? Pediatrics 116, 702-708.

31. Centuori S, Burmaz T, Ronfani L, Fragiacomo M, Quintero S, Pavan C, Davanzo R \& Cattaneo A (1999) Nipple care, sore nipples, and breastfeeding: a randomized trial. J Hum Lact $\mathbf{1 5}$, 125-130. 